\title{
FACTORES HUMANOS ASOCIADOS A LOS ACCIDENTES MORTALES DE MOTOCICLETAS SCOOTER EN ESPAÑA
}

\section{HUMAN FACTORS ASSOCIATED WITH FATAL MOTORCYCLE SCOOTER ACCIDENTS IN SPAIN}

\author{
María José Sospedra-Baeza* \\ Sergio Hidalgo-Fuentes** \\ Lia Cuñado-Pérez*** \\ *Facultad de Filosofía y Ciencias de la Educación, Universidad de Valencia. España \\ **Facultad de Psicología, Universidad de Valencia. España \\ ***Policía Local de Alboraya. España
}

\begin{abstract}
Resumen: Los accidentes de motocicleta son uno de los problemas de seguridad vial más importantes a nivel mundial. Hay una escasez de estudios específicos sobre las características de la accidentalidad de las motocicletas de tipo scooter, motivada por la falta de una definición precisa de este tipo de motocicleta y de la identificación de los distintos tipos de vehículos de dos ruedas en los registros de accidentes. El objetivo de este trabajo es determinar las características de los 293 conductores de motocicletas scooter fallecidos en España durante el periodo 2006-2011. Se realizaron análisis descriptivos y de asociación en función de la naturaleza de las variables. Los resultados de los análisis permiten identificar algunos de los principales factores humanos asociados a los accidentes mortales de los conductores de motocicletas scooter.
\end{abstract}

Palabras Clave: seguridad vial; motocicletas; factores de riesgo; accidentes de tráfico

Abstract: Motorcycle accidents are one of the most important road safety issues worldwide. There is a lack of specific researches about the characteristics of the scooter-motorcycle accidents, motivated by the absence not only of a precise definition and but also by a properly identification of the different type of two-wheeled vehicles within the accident's database. The aim of this study is to determine the characteristics of the 293 scooter-motorcycle drivers deceased in Spain in the period 2006 to 2011. According to the nature of the variables involved were performed descriptive and association analysis. The results of the analysis enable the identification some of the main human factors associated with the fatal accidents of scooter-motorcycle drivers.

Key Words: road safety; motorcycles; risk factors; motor traffic accidents

\section{Introducción}

Se estima que anualmente fallecen en el mundo 1.2 millones de personas y hasta 50 millones resultan heridas de diversa gravedad debido a accidentes de tráfico, lo que constituye la primera causa de mortalidad a nivel mundial entre jóvenes de entre 15 y 29 años (World Health Organization, 2015).

Sin embargo, la preocupación por este asunto no es nueva. La Organización Mundial de la Salud (OMS) publicó su primer informe relativo a los accidentes de tráfico y la seguridad vial en 1962 y, unos años más tarde, en 1974, la Asamblea Mundial de la OMS acordó la resolución WHA27.59, en la que se declaraba los accidentes de tráfico como un asunto de salud pública y exhortaba a los Estados miembros a abordar el problema (World Health Organization, 2008).
Los esfuerzos realizados en España, tanto por parte de la Dirección General de Tráfico (DGT) como de otras entidades que trabajan para reducir los accidentes de tráfico han dado sus frutos en los últimas décadas y han convertido a España en uno de los países de la Organización para la Cooperación y el Desarrollo Económicos (OECD) que más ha mejorado en los indicadores de seguridad vial, con una reducción de la tasa de mortalidad en este tipo de accidentes superior al $75 \%$ en los últimos 20 años (OECD, 2013).

España se encuentra actualmente a la cabeza de los países con menor tasa de mortalidad en accidentes de tráfico, con un total de 3.7 fallecidos cada 100.000 habitantes (World Health Organization, 2015).

Sin embargo, la reducción de la gravedad de los accidentes de tráfico en España durante los últimos años no se ha producido de manera homo-

Correspondencia: María José. Sospedra-Baeza. Facultad de Filosofía y Ciencias de la Educación, Universidad de Valencia. España. Correo electrónico: maria.jose.sospedra@uv.es 
génea entre los ocupantes de los distintos tipos de vehículos que conforman el parque móvil nacional, ya que, mientras los conductores y pasajeros de automóviles han reducido su mortalidad en más de un 75\% desde el año 2000 hasta el 2014 y los de ciclomotores (cilindrada no superior a $50 \mathrm{~cm}^{3}$ ) en casi un $90 \%$, los conductores y pasajeros de motocicletas (cilindrada superior a $50 \mathrm{~cm}^{3}$ )solo han reducido el número de muertos en accidentes de circulación en un $26.79 \%$, siendo la menor reducción de mortalidad en este periodo dentro de los vehículos a motor (Dirección General de Tráfico, 2015a). Además, la cifra de heridos hospitalizados a consecuencia de accidentes de tráfico ha aumentado en casi un $20 \%$ en este periodo, mientras que en el resto de vehículos motorizados se han experimentado importantes reducciones.

Esta situación no es exclusiva de España, sino que, como señala la International Road Traffic and Accident Database (IRTAD), en la gran mayoría de países la reducción del número de fallecidos en accidentes de tráfico en los últimos años es superior entre los ocupantes de automóviles que entre los de motocicleta (OECD/ITF, 2015).

No solo la reducción de la gravedad de los accidentes de motocicleta en España ha sido la que menor disminución ha registrado, sino que el peso de los accidentes de este tipo de vehículos dentro del total de accidentes de tráfico ha sufrido un aumento importante en los últimos años, pasando de haber al menos una motocicleta presente en un $11 \%$ de los accidentes con víctimas en 2004, a un 22\% en el 2013 (Dirección General de Tráfico, 2015b).

En los últimos años se ha producido asimismo un importante incremento del parque de motocicletas en España, pasando de un total de 1.445.644 motocicletas registradas en el año 2000 a un total de 2.972.165 en el 2014 (Dirección General de Tráfico, 2015c).

A nivel mundial, casi una cuarta parte de las muertes en accidentes de tráfico se dan entre motociclistas (World Health Organization, 2015).

Determinar los riesgos que incrementan la probabilidad de sufrir un accidente es una tarea compleja, ya que en la mayoría de los casos no es posible identificar un único factor como causa del accidente (Vlahogianni, Yannis, \& Golias, 2012). El factor humano está mucho más presente en los accidentes de ciclomotores y motocicletas que los factores relacionados con el vehículo o con la infraestructura y el entorno (Van Elslande et al., 2014).

Petridou y Moustaki (2000) presentaron una clasificación del factor humano en la conducción de vehículos diferenciando cuatro grandes grupos:
- Factores que reducen la capacidad base a largo plazo: inexperiencia, vejez, discapacidad, enfermedad, etc.

- Factores que reducen la capacidad base a corto plazo: somnolencia, fatiga, distracciones, conducir bajo los efectos del alcohol, etc.

- Factores que promueven comportamientos arriesgados con impacto a largo plazo: no uso del cinturón de seguridad o el casco, velocidad inadecuada de manera habitual, sobreestimación de las propias capacidades, etc.

- Factores que promueven comportamientos arriesgados con impacto a corto plazo: comportamiento suicida, drogas psicotrópicas, actos compulsivos, etc.

El uso de scooters se ha incrementado de manera ostensible en los últimos años en algunos países desarrollados, tanto en términos absolutos como relativos respecto al número de motocicletas (Blackman \& Haworth, 2013). Un importante porcentaje del parque de motocicletas en los países desarrollados son scooters, especialmente en zonas urbanas de algunos países europeos como España, Francia o Italia (Lin \& Kraus, 2009). Mientras que los conductores de motocicleta con más experiencia suelen ser propietarios de motocicletas de alto rendimiento, los nuevos conductores se decantan por modelos tipo scooter de baja cilindrada (Jamson \& Chorlton, 2009).

Sin embargo, debido a la falta de una definición precisa y a que, por lo general, en los registros de datos de accidentes no se identifica el tipo de motocicleta implicada (los scooters únicamente se clasifican dentro del grupo de ciclomotores o motocicletas, dependiendo de su cilindrada), no se han realizado muchos estudios comparativos del riesgo entre ciclomotores y/o motocicletas y scooters (Blackman \& Haworth, 2013).

Por todo ello, la finalidad del estudio es determinar los factores humanos relacionados con la accidentalidad mortal de los conductores de motocicletas de tipo scooter que posibilite desarrollar a partir de ello, programas de prevención, debido a la escasez de investigaciones anteriores sobre el tema.

\section{Método}

El estudio se realizó sobre el total de accidentes mortales de motocicletas scooter ocurridos en España desde el año 2006 hasta el año 2011. El 92.5 de estos conductores eran varones, mientras que el 7.5 eran mujeres. La edad media era de 41.06 años $(D E=12.47)$. 
Se trata de un estudio de observación descriptivo exploratorio, retrospectivo y transversal de todos los accidentes mortales de motocicleta tipo scooter ocurridos entre los años 2006 - 2011. Los datos utilizados para este estudio provienen de la base de datos de accidentes con víctimas de la DGT correspondientes a dicho periodo.

La información de los registros incluidos en esta base es recogida directamente por los agentes encargados de la vigilancia y control del tráfico en el momento del accidente mediante un cuestionario estadístico en el que se registran los datos propios del accidente, de los vehículos implicados y de las personas que se han visto involucradas.

El tipo de motocicleta implicada en el accidente no se registra en dicho cuestionario con fines estadísticos. Por ello, se generó una nueva variable en la base de datos con la clasificación de las motocicletas (scooter/no scooter) siguiendo los criterios del Departamento de Transporte de los EEUU (Motorcycle controls and displays, 2011), que son los siguientes:

- Cuenta con una plataforma para los pies del conductor o con reposapiés integrados.

- La parte de la motocicleta situada delante del asiento del conductor es de menor altura que el asiento del conductor.

Después de depurar las bases de datos y seleccionar aquellos accidentes en los que había fallecido al menos un ocupante de motocicleta tipo scooter quedó como resultado una base de datos con un total de 293 conductores de motocicletas scooter fallecidos en accidente de tráfico durante el periodo comprendido entre los años 2006 y 2011.

Los datos fueron sometidos a análisis descriptivos y de asociación (Chi-cuadrado y Eta); se realizaron contrastes de hipótesis de correlación $\left(H_{0}: \rho_{x y}=0\right.$ y $\left.H_{1:} \rho_{x y} \neq 0\right)$ tetracórica, biserial puntual, en función de la naturaleza de las variables.

Se elaboraron diagramas de caja y gráficos de frecuencias, utilizando el paquete estadístico SPSS 22.0.

\section{Resultados}

El mayor porcentaje de conductores fallecidos tenían en el momento del accidente entre 25 y 44 años (56.9\%), con una edad media de 41.06 años $(D E=12.47)$. En la mayoría de los accidentes (94.1\%), el conductor circulaba solo (sin pasajero).

El 92.5\% de los conductores de motocicletas scooter fallecidos en accidentes de tráfico entre los años 2006 y 2011 en España fueron hombres (ver tabla 1) con una edad media de 41.6 años $(D E=12.5)$; en el caso de las mujeres conductoras la media de edad fue de 34.41 años $(D E=10.6)$. Hay que considerar que el porcentaje de hombres conductores de motocicletas scooter que en este periodo se vio involucrado en algún accidente (independientemente de la gravedad) fue del $82.6 \%$, mientras que en el caso de las mujeres dicho porcentaje fue del $17.4 \%$,

Tabla 1

Media de edad de los conductores de motocicletas scooter fallecidos por sexo

\begin{tabular}{lcccc}
\hline Sexo & Frecuencia & $M$ & $D E$ & $\begin{array}{c}\text { Error } \\
\text { estándar }\end{array}$ \\
\hline Hombre & 92.5 & 41.60 & 12.46 & .761 \\
Mujer & 7.5 & 34.41 & 10.63 & 2.267 \\
\hline Total & 100 & 41.06 & 12.47 & .732 \\
\hline
\end{tabular}

El principal motivo de desplazamiento en este tipo de accidentes fue por ocio (44.6\%), aunque también hubo un porcentaje considerable (27.93\%) que tuvo lugar en desplazamientos por motivos laborales (in itinere o en misión) (ver figura 1).

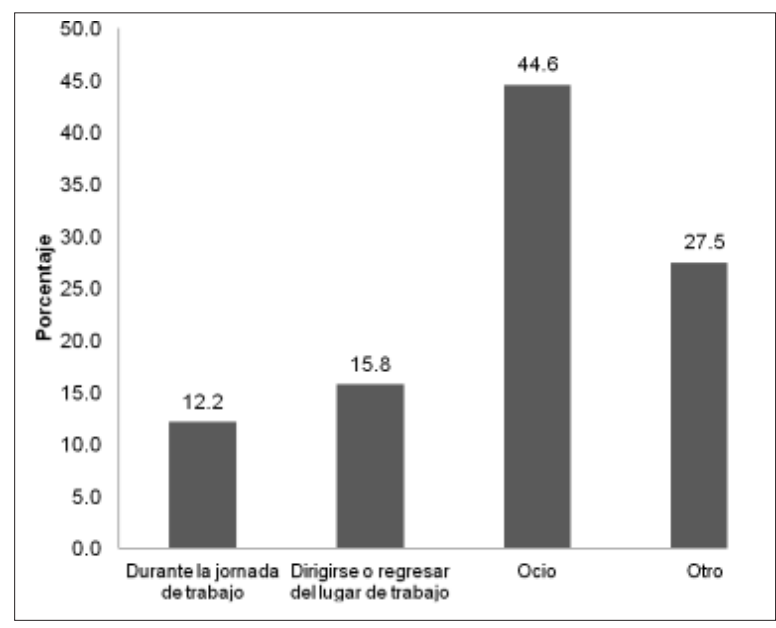

Figura 1. Motivo del desplazamiento en el momento del accidente.

En la mayoría de los accidentes mortales (93.7\%) no concurrieron condiciones psicofísicas negativas (alcohol, drogas, sueño y cansancio). Solo en el 3.2\% de ellos los conductores presentaban síntomas relacionados con la ingesta de alcohol.

En cuanto a las infracciones de conducción identificadas en la base de datos de la DGT, el mayor porcentaje de las mismas fueron de distracción (22.59\%), seguidas de la invasión parcial del sentido contrario y de adelantamientos antirreglamentarios (4.44\% en ambos casos) y de no cumplir la señal de Stop (3.33\%). Tan solo el $27.04 \%$ de los conductores fallecidos no cometieron ninguna infracción de conducción en el momento del accidente. 
En el $42.4 \%$ de los accidentes mortales de motocicletas scooter se produjo una infracción de velocidad, bien sobrepasar la velocidad establecida (10.4\%), bien circular a una velocidad inadecuada para las condiciones existentes (31.9\%).

En relación a las infracciones administrativas, el 9.6\% cometió algún tipo de infracción administrativa (un $8.5 \%$ no tenía el permiso de conducción adecuado y un $1.1 \%$ no había pasado la Inspección Técnica de Vehículos) (ver tabla 2).

Tabla 2

Principales infracciones cometidas en el momento del accidente

\begin{tabular}{lcc}
\hline Infracciones & & Porcentaje \\
\hline Administrativas & $\begin{array}{c}\text { Carecer del permiso de } \\
\text { conducción adecuado } \\
\text { No haber pasado la } \\
\text { Inspección Técnica } \\
\text { Reglamentaria del } \\
\text { Vehículo }\end{array}$ & 8.5 \\
& $\begin{array}{c}\text { Distracción } \\
\text { Conducción }\end{array}$ & 22.6 \\
& $\begin{array}{c}\text { Invadir parcialmente el } \\
\text { sentido contrario } \\
\text { Adelantar }\end{array}$ & 4.4 \\
& $\begin{array}{c}\text { antirreglamentariamente } \\
\text { No cumplir las señal } \\
\text { de Stop }\end{array}$ & 4.4 \\
\hline Velocidad & $\begin{array}{c}\text { Exceso de velocidad } \\
\text { Velocidad inadecuada }\end{array}$ & 10.4 \\
\hline
\end{tabular}

Se obtuvo el coeficiente de correlación biserial puntual (rbp) entre la variable utilización del casco y edad ( $r b p=-0.046 ; p=.433)$, no se detectó relación estadísticamente significativa entre ambas variables. La media de edad de los sujetos que utilizaban y no utilizaban el casco en el momento del accidente (ver figura 2) es muy similar, sin embargo, la variabilidad entre las edades es mayor en el caso de los que sí lo usaban.

Tampoco se obtuvo relación estadísticamente significativa entre la utilización del casco y el sexo. En este caso el coeficiente aplicado fue el de correlación tetracórica $(r t=0.07 ; p=.264)$.

El análisis de asociación entre la utilización del casco y las infracciones administrativas y de conducción se realizó mediante la prueba Chi cuadrado y el coeficiente de contingencia. Dado que en ambos casos más del $20 \%$ de las casillas tenía frecuencias esperadas menores que 5, la probabilidad asociada a Chi cuadrado se obtuvo mediante el método de Montecarlo.

No se detectó ninguna asociación estadísticamente significativa entre el uso del casco y la comisión de infracciones de ambos tipos (administrativas o de conducción) en el momento del accidente. A pesar de ello, merece la pena destacar que el $20 \%$

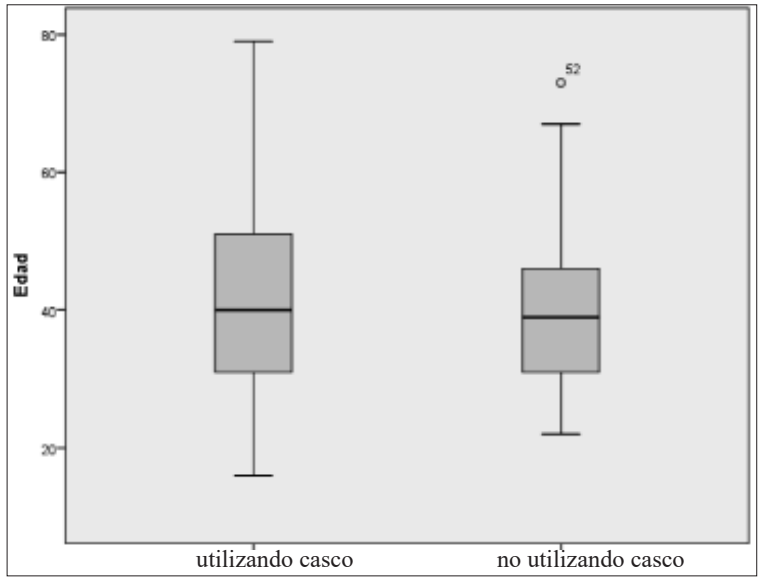

Figura 2. Diagrama de caja del uso del casco por edad.

de los fallecidos que no utilizaban el casco en el momento del accidente, carecían del permiso de conducción adecuado y que el $23.35 \%$ de los sujetos que cometieron algún tipo de infracción de conducción (mayoritariamente de distracción) no utilizaban el casco en el momento del accidente.

Tampoco se detectó una asociación estadísticamente significativa entre la utilización del casco y el motivo del desplazamiento.

En cuanto a la edad de los conductores fallecidos, existe una asociación media con las infracciones de conducción $(\mathrm{Eta}=0,641)$. Los conductores que en el momento del accidente cometieron una infracción consistente en no respetar una señal de Stop fueron más jóvenes que los que cometieron el resto de infracciones (ver figura 3 ), mientras que los que cometieron una infracción relacionada con la distracción fueron los de mayor edad, aunque estas diferencias no resultaron estadísticamente significativas.

En relación al sexo, no se han detectado diferencias estadísticamente significativas en el tipo de infracción de conducción que cometieron hombres y mujeres. En ambos casos (el 67.5\% de hombres y el $50 \%$ de mujeres) la infracción mayoritaria fue la distracción.

Por lo que respecta al motivo del desplazamiento se ha detectado que existe relación estadísticamente significativa entre esta variable y las infracciones de conducción cometidas por los conductores de motocicleta scooter en el momento del accidente mortal $\left(\chi_{(9)}^{2}=28,92 ; p=.002\right)$. La significación asociada al estadístico se ha obtenido mediante el método Monte Carlo dado que no se cumplían los criterios de obtención de la probabilidad asintótica, obteniéndose un valor de probabilidad para comprobar la significación estadística de .002 (ver tabla 3). 


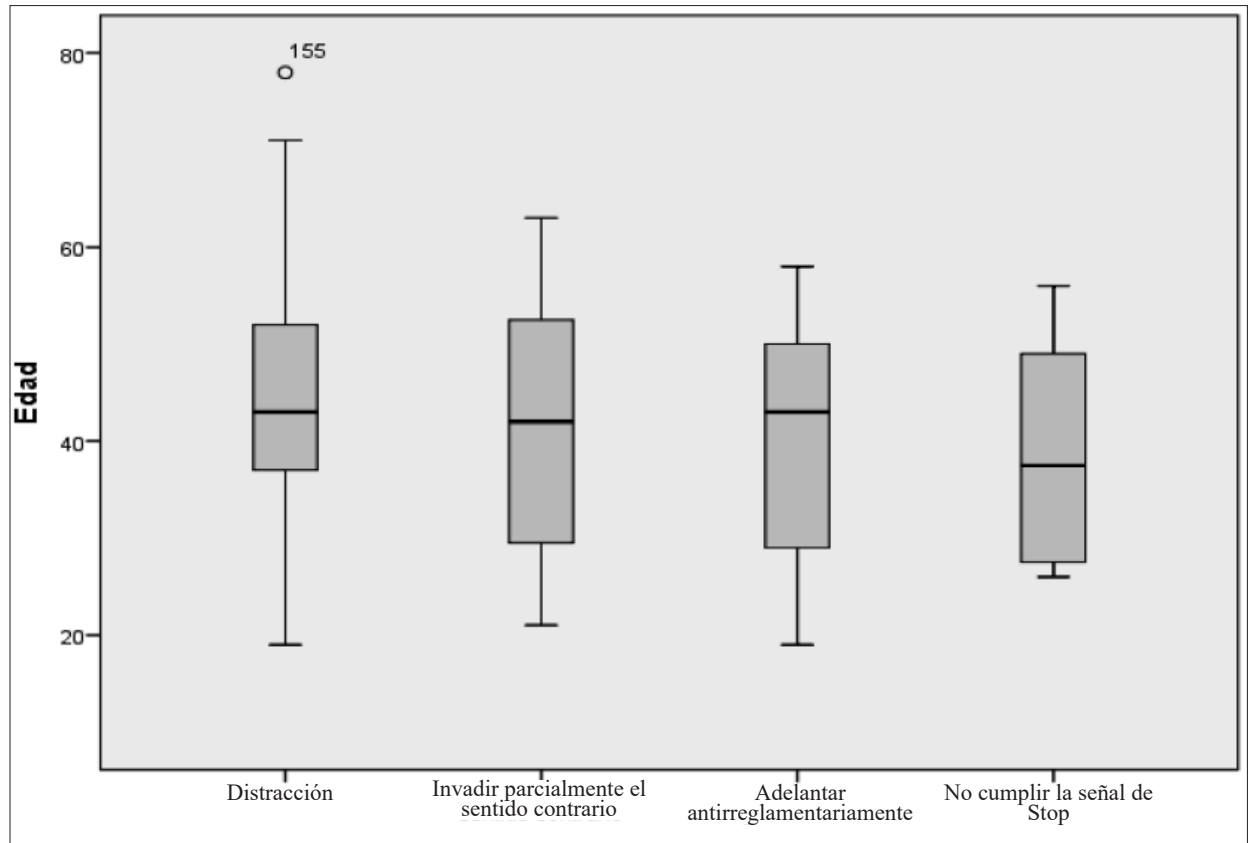

Figura 3. Diagrama de caja de infracciones de conducción por edad.

Atendiendo a los residuos ajustados que tienen una distribución normal con $\mu=0$ y $\sigma=1$, observamos que la asociación significativa (residuos mayores a \pm 1.96 ) se obtiene para las infracciones distracción y no parar en un Stop durante la jornada de trabajo (ver tabla 4).

En el $99 \%$ de los casos la probabilidad exacta obtenida por el método Monte Carlo se sitúa entre .001 y .003. La asociación entre las variables alcanza un nivel medio de significación (coeficiente de contingencia $=0.508$ ). Atendiendo al valor de los residuos corregidos, se puede concluir que la mayoría de los accidentes mortales entre los conductores de motocicleta scooter son debidos a no respetar la señal de Stop durante la jornada de trabajo.

Para analizar la existencia de relación entre el uso o no uso del casco y las variables edad, sexo, infracciones de conducción, infracciones administrativas y motivo de desplazamiento se obtuvieron distintos coeficientes de correlación e índices de asociación en función de la naturaleza de las variables.

Tabla 3.

Chi-cuadrado. Infracciones de conducción por motivo de desplazamiento

\begin{tabular}{|c|c|c|c|c|}
\hline & \multirow{3}{*}{ Valor } & \multicolumn{3}{|c|}{ Significación de Monte Carlo } \\
\hline & & \multirow{2}{*}{ Significación } & \multicolumn{2}{|c|}{ Intervalo de confianza al 99\% } \\
\hline & & & Límite inferior & Límite superior \\
\hline $\begin{array}{l}\text { Coeficiente de } \\
\text { contingencia }\end{array}$ & .508 & $.002^{\mathrm{c}}$ & .001 & .003 \\
\hline $\begin{array}{l}\mathrm{N} \text { de casos } \\
\text { válidos }\end{array}$ & 83 & & & \\
\hline
\end{tabular}

Tabla 4. Residuos ajustados infracciones de conducción por motivo de desplazamiento

\begin{tabular}{cccccc}
\hline & & \multicolumn{3}{c}{ infracciones de conducción } \\
\cline { 3 - 5 } & & Distracción & $\begin{array}{c}\text { Invasión } \\
\text { sentido } \\
\text { contrario }\end{array}$ & $\begin{array}{c}\text { Adelantamiento } \\
\text { antirreglamentario }\end{array}$ & No Stop \\
\hline $\begin{array}{c}\text { Motivo } \\
\text { del desplazamiento }\end{array}$ & $\begin{array}{c}\text { Durante la } \\
\text { jornada de } \\
\text { trabajo } \\
\text { Dirigirse o } \\
\text { regresar del } \\
\text { lugar de trabajo }\end{array}$ & -2.0 & -1.0 & 1.7 & 4.0 \\
Ocio & 1.4 & -1.7 & .3 & -.8 \\
Otro & 1.7 & -1.8 & 2.6 & -.8 & .0 \\
\hline
\end{tabular}




\section{Discusión}

El perfil del conductor de motocicleta scooter fallecido en accidente en España desde 2006 hasta 2011 es de un varón de 41.6 años de edad con permiso de conducir de la clase A o B, que circula sin acompañante en desplazamientos de ocio en condiciones psicofísicas óptimas, y que comete alguna infracción de conducción, fundamentalmente de distracción.

El hecho de que los conductores de edad media sean los que más frecuentemente se ven involucrados en accidentes mortales no está en consonancia con la literatura científica analizada, que señala que los conductores de edad avanzada y los más jóvenes presentan un mayor riesgo de verse involucrados en accidentes de tráfico (Augenstein, Perdeck, Stratton, Digges, \& Bahouth, 2003; Kim, Ulfarsson, Kim \& Shankar, 2013; Li, Braver, \& Chen, 2003; Lyman, Ferguson, Braver, \& Williams, 2002; Newgard, 2008). En el caso específico de conductores de motocicleta, diversos autores también apuntan a la juventud como un factor de riesgo (Mullin, Jackson, Langley, \& Norton, 2000; Rutter \& Quine, 1996; Ulleberg, 2003; Yeh \& Chang, 2009), sin embargo, es importante señalar que debido a la imposibilidad de poder relacionar el número de conductores accidentados con el número total de conductores de motocicletas scooter, no es posible analizar el riesgo de cada grupo de edad. Así pues, la causa de un mayor número de accidentes en determinados grupos de edad en el periodo analizado puede deberse a que en dichos grupos se concentra un mayor número de conductores y no a que estos grupos se ven expuestos a un mayor riesgo.

El hecho de que un porcentaje muy importante de los conductores de motocicletas de tipo scooter fallecidos en el periodo analizado sean varones concuerda con el trabajo de Lin, Chang, Pai y Keyl (2003) según el cual los conductores de motocicleta varones jóvenes muestran una mayor propensión a adoptar comportamientos de riesgo, lo que se asocia a una mayor probabilidad de verse involucrado en un accidente.

Los accidentes de tráfico se encuentran a menudo relacionados con el consumo de alcohol $\mathrm{u}$ otras sustancias tóxicas (Compton, Blomberg, Moscowitz, Burns, Peck, \& Fiorentino, 2002; Kelly, Darke, \& Ross, 2004; Petridou \& Moustaki, 2000; Raes, Van den Neste, Verstraete, Lopez, Hughes, \& Griffiths, 2008; Walsh, Gier, Christopherson, \& Verstraete, 2004). En el período analizado, el 1.4\% de los conductores de motocicletas de tipo scooter accidentados se encontraban bajo los efectos del alcohol y/u otras drogas a juicio de los agentes encargados de la vigilancia y control del tráfico. Este dato podría deberse a que, como apuntan diversos estudios (Moskal, Martin, \& Laumon, 2012; Sexton, Baughan, Elliott, \& Maycock, 2004), las motocicletas de tipo scooter suelen ser utilizadas de manera más frecuente para desplazamientos relacionados con asuntos laborales y menos para propósitos recreativos o de ocio, donde sería más probable que el conductor consumiera bebidas alcohólicas $u$ otras sustancias. Además, el consumo de alcohol se encuentra más extendido entre los conductores de motocicleta más jóvenes (Holubowycz, Kloeden, \& McLean, 1994; Zambon \& Hasselberg, 2006), y la media de edad de los conductores de motocicletas de tipo scooter fallecidos durante este periodo es más elevada (41.06 años).

La velocidad es uno de los comportamientos de riesgo más comunes tanto en la producción de accidentes de tráfico como en la gravedad de sus consecuencias ya sea de manera general (Ellison \& Greaves, 2015; Elvik, Christensen, \& Amundsen, 2004) como de manera específica en la accidentalidad de vehículos de dos ruedas (Lardelli-Claret, Jimenez-Moleon, Luna-del-Castillo, García-Martín, Bueno-Cavanillas, \& Gálvez-Vargas, 2005; Steg \& Brussel, 2009).

A pesar de todas las campañas que se vienen realizando por instituciones y organismos tanto públicos como privados para concientizar sobre la importancia del uso del casco, el $14 \%$ de los conductores de scooter fallecidos en España entre los años 2006 y 2011 no hacían uso del casco en el momento del accidente. Numerosas investigaciones señalan como la utilización de este dispositivo de seguridad reduce el riesgo de lesiones en la cabeza y la cara, así como de fallecimiento en caso de accidente (Liu, Ivers, Norton, Boufous, Blows, \& Lo, 2008; Moskal, Martin, \& Laumon, 2008; Rowland, Rivara, Salzberg, Soderberg, Maier, \& Koepsell, 1996).

Un $8.5 \%$ de los conductores fallecidos no contaba en el momento del accidente con el permiso de conducir adecuado, circunstancia que aumenta de riesgo de verse involucrado en un accidente frente a aquellos que sí conducen con un permiso válido (Moskal et al., 2012). Este mayor riesgo entre conductores de motocicleta que conducen sin un permiso válido también ha sido observado en otros estudios (Lardelli-Claretet al., 2005; Lin, Chang, Pai, \& Keyl, 2003).

Es una realidad la accidentalidad de los vehículos de dos ruedas, pero su prevención no pasa por intentar reducir su uso sino por implementar 
medidas de prevención, basadas fundamentalmente en lograr un cambio de actitud de los conductores, el entrenamiento no debe centrarse únicamente en habilidades básicas de conducción y el dominio de las situaciones de tráfico, sino dirigirse a las actitudes hacia la seguridad, poniendo un especial énfasis, en la percepción de riesgo y la conducción defensiva (Kardamanidis, Martiniuk, Ivers, Stevenson, \& Thistlethwaite, 2010) y no solo debe ir dirigido a los conductores de los vehículos de dos ruedas sino que todos los usuarios de la vía deben conocer y ser conscientes de la vulnerabilidad de los vehículos de dos ruedas. No hay que olvidar que la vía es compartida por todos y es fundamental modificar las actitudes de todos los que conviven en ella, por lo que la mejor estrategia para abordar la accidentalidad de estos vehículos es hacerlo desde un enfoque global que integre a todos los usuarios de la vía, dentro de los planes de movilidad y las estrategias de seguridad (Van Elslande et al., 2014).

Sería importante que los organismos responsables del control y la vigilancia del tráfico recogieran el tipo y la cilindrada de las motocicletas implicadas en accidentes de circulación, ya que a la hora de estudiar la accidentalidad de motocicletas sería muy útil establecer comparaciones entre sus distintos tipos.

También se recomienda que en los datos de matriculaciones y parque de vehículos se distinga entre los diferentes tipos de motocicleta ya que, si queremos llevar a cabo análisis comparativos entre ellas, es necesario, además del número de accidentes, vehículos y personas implicadas, calcular tasas en función del número de motocicletas de cada tipo matriculadas dentro del ámbito que estemos estudiando.

Como hemos comentado anteriormente, no existen apenas investigaciones sobre la accidentalidad de las motocicletas tipo scooter a pesar del importante aumento del peso de este tipo de vehículos dentro del parque de motocicletas. Por tanto, se recomienda continuar con el estudio de las características de los accidentes de esta clase de motocicletas, así como de las personas accidentadas, con el objetivo de dotar a los agentes decisores de la información necesaria para la planificación, la implantación y el desarrollo de las medidas necesarias para reducir la accidentalidad de las mismas.

La principal limitación de este trabajo emana de la fuente de datos utilizada, el Registro de accidentes de la DGT. La depuración de las tres bases de datos puso de manifiesto un gran número de errores y datos faltantes en diversas variables, especialmente en la codificación del tipo de vehículo.
En lo referente a las motocicletas implicadas en accidentes de circulación durante el periodo analizado, el Registro de accidentes de la DGT no distingue ni el tipo de motocicleta, ni la cilindrada de la misma, lo que impide llevar a cabo análisis comparativos más profundos. El 1 de enero de 2015 comenzó a utilizarse un nuevo Formulario de Accidentes con Víctimas en el que, aunque sí se recoge la cilindrada (inferior o superior a los $125 \mathrm{~cm}^{3}$ ), no se clasifican las motocicletas según la tipología, lo que dificulta la realización de estudios como el presente.

\section{Referencias}

Augenstein, J., Perdeck, E., Stratton, J., Digges, K., \& Bahouth, G. (2003). Characteristics of Crashes that Increase the Risk of Serious Injuries. AnnualProceedings / AssociationfortheAdvancement of Automotive Medicine, 47, 561-576.

Blackman, R. A., \& Haworth, N. L. (2013). Comparison of moped, scooter and motorcycle crash risk and crash severity. Accident Analysis \& Prevention, 57, 1-9. doi:10.1016/j. aap.2013.03.026

Compton, R. P., Blomberg, R. D., Moscowitz, H., Burns, M., Peck, R. C., \& Fiorentino, D. D. (2002). Crash risk of alcohol impaired driving. Proceedings International Council on Alcohol, Drugs and Traffic Safety Conference (Vol. 2002, pp. 39-44). International Council on Alcohol, Drugs and Traffic Safety.

Dirección General de Tráfico (2015a). Anuario Estadístico de Accidentes. 2014. Ministerio del Interior. Dirección General de Tráfico, Madrid.

Dirección General de Tráfico (2015b). Las principales cifras de la siniestralidad vial. España 2014. Ministerio del Interior. Dirección General de Tráfico, Madrid.

Dirección General de Tráfico (2015c). Anuario Estadístico General. 2014. Ministerio del Interior. Dirección General de Tráfico, Madrid.

Ellison, A. B., \& Greaves, S. P. (2015). Speeding in urban environments: are the time savings worth the risk? Accident Analysis \& Prevention, 85, 239-247. doi:10.1016/j. aap.2015.09.018

Elvik, R., Christensen, P., \& Amundsen, A. (2004). Speed and road accidents: An evaluation of the Power Model. TOI report 740 (Vol. 740).

Holubowycz, O. T., Kloeden, C. N., \& McLean, A. J. (1994). Age, sex, and blood alcohol concentration of killed and injured drivers, riders, and passengers. Accident Analysis \& Prevention, 26(4), 483-492. doi:10.1016/00014575(94)90039-6

Jamson, S., \& Chorlton, K. (2009). The changing nature of motorcycling: Patterns of use and rider characteristics. Transportation research part F: traffic psychology and behaviour, 12(4), 335-346. doi:10.1016/j.trf.2009.04.002

Kardamanidis, K., Martiniuk, A., Ivers, R.Q., Stevenson, M.R., \& Thistlethwaite, K. (2010). Motorcycle rider training for the prevention of road traffic crashes. Cochrane Database of Systematic Reviews, 10, CD005240. doi:10.1002/14651858.CD005240.pub2

Kelly, E., Darke, S., \& Ross, J. (2004). A review of drug use and driving: epidemiology, impairment, risk factors and risk perceptions. Drug and alcohol review, 23(3), 319-344. doi:10.1080/09595230412331289482 
Kim, J. K., Ulfarsson, G. F., Kim, S., \& Shankar, V. N. (2013). Driver-injury severity in single-vehicle crashes in California: a mixed logit analysis of heterogeneity due to age and gender. Accident Analysis \& Prevention, 50, 1073-1081. doi:10.1016/j.aap.2012.08.011

Lardelli-Claret, P., Jimenez-Moleon, J. J., de Dios Luna-delCastillo, J., García-Martín, M., Bueno-Cavanillas, A., \& Gálvez-Vargas, R. (2005). Driver dependent factors and the risk of causing a collision for two wheeled motor vehicles. Injury Prevention, 11(4), 225-231. doi:10.1136/ ip.2004.006957

Li, G., Braver, E. R., \& Chen, L. H. (2003). Fragility versus excessive crash involvement as determinants of high death rates per vehicle-mile of travel among older drivers. Accident Analysis \& Prevention, 35(2), 227-235. doi:10.1016/s0001-4575(01)00107-5

Lin, M. R., Chang, S. H., Pai, L., \&Keyl, P. M. (2003). A longitudinal study of risk factors for motorcycle crashes among junior college students in Taiwan. Accident Analysis \& Prevention, 35(2), 243-252. doi:10.1016/ s0001-4575(02)00002-7

Lin, M. R., \&Kraus, J. F. (2009). A review of risk factors and patterns of motorcycle injuries. Accident Analysis \& Prevention, 41(4), 710-722. doi:10.1016/j.aap.2009.03.010

Liu, B. C., Ivers, R., Norton, R., Boufous, S., Blows, S., \& Lo, S. K. (2008). Helmets for preventing injury in motorcycle riders. Cochrane Database Systematic. Reviews, 2008 (1), CD004333. doi: 10.1002/14651858.CD004333.pub3

Lyman, S., Ferguson, S. A., Braver, E. R., \& Williams, A. F. (2002). Older driver involvements in police reported crashes and fatal crashes: trends and projections. Injury prevention, 8(2), 116-120.doi:10.1136/ip.8.2.116

Moskal, A., Martin, J. L., \&Laumon, B. (2008). Helmet use and the risk of neck or cervical spine injury among users of motorized two-wheel vehicles. Injury Prevention, 14(4), 238-244.doi:10.1136/ip.2007.018093

Moskal, A., Martin, J. L., \&Laumon, B. (2012). Risk factors for injury accidents among moped and motorcycle riders. Accident Analysis \& Prevention, 49, 5-11. doi:10.1016/j. aap.2010.08.021

Motorcycle controls and displays, 49 CFR 571.123 - Standard No. 123 (2011).

Mullin, B., Jackson, R., Langley, J., \& Norton, R. (2000). Increasing age and experience: are both protective against motorcycle injury? A case-control study. Injury Prevention, 6(1), 32-35.

Newgard, C. D. (2008). Defining the "older" crash victim: The relationship between age and serious injury in motor vehicle crashes. Accident Analysis \& Prevention, 40(4), 1498-1505. doi:10.1016/j.aap.2008.03.010

OECD (2013). Health at a Glance 2013: OECD Indicators. OECD Publishing. doi: 10.1787/health_glance-2013-en
OECD/ITF (2015), Road Safety Annual Report 2015. OECD Publishing, Paris. doi:10.1787/irtad-2015-en

Petridou, E., \& Moustaki, M. (2000). Human factors in the causation of road traffic crashes. European journal of epidemiology, 16(9), 819-826.

Raes, E., Van den Neste, T., Verstraete, A., Lopez, D., Hughes, B., \& Griffiths, P. (2008). Drug use, impaired driving and traffic accidents (Vol. 8). EMCDDA.

Rowland, J., Rivara, F., Salzberg, P., Soderberg, R., Maier, R., \& Koepsell, T. (1996). Motorcycle helmet use and injury outcome and hospitalization costs from crashes in Washington State. American Journal of Public Health, 86(1), 41-45. doi:10.1016/s0736-4679(96)89805-2

Rutter, D. R., \& Quine, L. (1996). Age and experience in motorcycling safety. Accident Analysis \& Prevention, 28(1), 15-21. doi:10.1016/0001-4575(95)00037-2

Sexton, B., Baughan, C., Elliott, M, \& Maycock, G. (2004) The accident risk of motorcyclists. Crowthorne: Transport Research Laboratory.

Steg, L., \&Brussel, A. V. (2009). Accidents, aberrant behaviours, and speeding of young moped riders. Transportation research part F: traffic psychology and behaviour, 12(6), 503-511. doi:10.1016/j.trf.2009.09.001

Ulleberg, P. (2003). Motorcycle safety-A literature review and meta-analysis. Oslo: Institute of Transport Economics, Norway.

Van Elslande, P., Feypell-de La Beaumelle, V., Holgate, J., Redant, K., De Solere, H., Margaritis, D., \&Granström, P. O. (2014, April). Mobility and safety of powered twowheelers in the OECD countries. In TRA2014 Transport Research Arena: Transport Solutions: from Research to Deployment-Innovate Mobility, Mobilise Innovation! Paris, pp.1-11.

Vlahogianni, E. I., Yannis, G., \& Golias, J. C. (2012). Overview of critical risk factors in Power-Two-Wheeler safety. Accident Analysis \& Prevention, 49, 12-22. doi:10.1016/j. aap.2012.04.009

Walsh, J. M., Gier, J. J., Christopherson, A. S., \& Verstraete, A. G. (2004). Drugs and driving. Traffic injury prevention, 5(3), 241-253. doi:10.1080/15389580490465292

World Health Organization. (2008). World report on road traffic injury prevention, 2004. World Health Organization: Geneva.

World Health Organization. (2015). Global status report on road safety 2015. World Health Organization: Geneva.

Yeh, T. H., \& Chang, H. L. (2009). Age and contributing factors to unlicensed teen motorcycling. Safety science, 47(1), 125-130. doi:10.1016/j.ssci.2008.02.002

Zambon, F., \& Hasselberg, M. (2006). Factors affecting the severity of injuries among young motorcyclists a Swedish nationwide cohort study. Traffic injury prevention, 7(2), 143-149. doi:10.1080/15389580600555759

Para citar este artículo:

Sospedra-Baeza, M. J., Hidalgo-Fuentes, S., \& Cuñado-Pérez, L. (2017). Factores humanos asociados a los accidentes mortales de motocicletas scooter en España. Ciencias Psicológicas, 11(1), 49 - 56. doi: 10.22235/cp.v11i2.1346 